\title{
Aqueous Dispersions of Graphene from Electrochemically Exfoliated Graphite
}

\author{
Marta Sevilla, Guillermo A. Ferrero and Antonio B. Fuertes* \\ Instituto Nacional del Carbón (CSIC), P.O. Box 73, Oviedo 33080, Spain \\ *Corresponding author: abefu@incar.csic.es
}

\begin{abstract}
This work presents a facile and environmentally-friendly synthesis strategy for the production of stable and easily processable dispersions of graphene in water. This strategy represents an alternative to the classical chemical exfoliation methods (v.g. Hummers' method), which are more complex, harmful and dangerous. The process is based on the electrochemical exfoliation of graphite and includes three simple steps: (a) the anodic exfoliation of graphite in $\left(\mathrm{NH}_{4}\right)_{2} \mathrm{SO}_{4}$, (b) sonication to separate the oxidized graphene sheets and (c) reduction of the oxidized graphene to graphene. The procedure involves relatively short processing times and allows high yields, making it possible to convert around 30 wt.\% of the initial graphite into graphene. The graphene sheets are well-dispersed in water, have a $\mathrm{C} / \mathrm{O}$ atomic ratio of 11.7 , a lateral size of $\sim 0.5-1 \mu \mathrm{m}$ and they contain only a few graphene layers, most of which are bilayer sheets. The processability of this type of aqueous dispersion has been demonstrated in the fabrication of macroscopic graphene structures, such as graphene aerogels and graphene films, which have been successfully employed respectively as absorbents or as electrodes in supercapacitors.
\end{abstract}

Keywords: Graphene, graphite, electrochemical exfoliation, aqueous dispersion. 


\section{Introduction}

The unique electronic, thermal, chemical, optical and mechanical properties of graphene make it a promising material for a variety of applications in electronics, optical devices, high-performance composites, energy storage and conversion systems, field emission devices and sensors. ${ }^{[1]}$ The graphene can be produced by different methods based on either bottom-up (e.g. chemical vapor deposition (CVD), arc discharge, etc.) or top-down (e.g. physical and chemical exfoliation of graphite) synthesis strategies. Although CVD and other bottom-up methods can produce a high-quality graphene, these processes are inviable for scalingup because of their high cost and complexity. In contrast, top-down procedures based on the liquid-phase exfoliation of graphite constitute a reliable route for manufacturing graphene on a large scale. The direct liquid-phase exfoliation of graphite (physical exfoliation) can be carried out by means of ultrasonication or shear mixing in organic solvents (i.e. NMP or DMF) or in aqueous solutions in the presence of surfactants. ${ }^{[2]}$ However, these procedures suffer from longer exfoliation times, low yields and the need for toxic solvents, factors which hinder the scaling-up of the process for the production of large amounts of graphene. Until now, the chemical exfoliation of graphite based on the Hummers method and analogous procedures have been the most popular route for producing graphene oxide (GO), which can be converted to graphene by reduction following a variety of procedures. Importantly, in $2008 \mathrm{Li}$ et al. proposed a method of fabricating highly stable aqueous dispersions of graphene nanosheets based on the "in situ" reduction of GO suspensions. ${ }^{[3]}$ This type of aqueous dispersion is highly versatile, easily processable and, in consequence, offers a sound basis for producing a large variety of graphene-based materials 
useful for a wide range of potential applications. ${ }^{[4]}$ However, the major drawback of this synthesis strategy lies in the fact that the production of $\mathrm{GO}$ is based on the Hummers method which entails several considerable risks (i.e. manipulation of highly corrosive compounds, the emission of toxic gases, the generation of potentially explosive compounds and the production of a large volume of contaminant residues), limiting its scalability. ${ }^{[5]}$

Recently, the electrochemical exfoliation of graphite (EEG) has emerged as a reliable option for producing graphene sheets with a large lateral size, a good conductivity and a high transparency. Compared to other exfoliation approaches, this process has the advantage that it is simple, one-step, low-cost, environmentally friendly and potentially scalable. ${ }^{[6]}$ Although EEG can be carried out by using non-aqueous electrolytes (i.e. alkylammonium salts dissolved in organic solvents) and graphite as the cathode, from a practical point of view it is more convenient to use graphite as the anode and an aqueous electrolyte, which typically consists of acids (e.g. $\mathrm{H}_{2} \mathrm{SO}_{4}, \mathrm{H}_{3} \mathrm{PO}_{4}$, etc.) ${ }^{[7]}$ or inorganic salts (e.g. $\mathrm{Na}_{2} \mathrm{SO}_{4},\left(\mathrm{NH}_{4}\right)_{2} \mathrm{SO}_{4}, \mathrm{~K}_{2} \mathrm{SO}_{4}$, etc.). ${ }^{[8]}$ The properties of the graphene sheets obtained by EEG depends on the type of electrolyte and its concentration, in addition to the characteristics of the starting graphite and the operation conditions used in the EEG process, such as electrolysis time or voltage. ${ }^{[9]}$ Usually, the above parameters are optimized to obtain high-quality graphene layers with low oxygen contents: C/O atomic ratio values in the 9-26 range. $^{[10]}$ Unfortunately, graphene sheets obtained in this way have a hydrophobic nature and therefore cannot be dispersed in water, which makes their processing difficult. Alternatively, they can be dispersed in organic toxic solvents of high boiling point such as DMF (N,N-dimethylformamide) or NMP 
(N-methyl-2-pyrrolidone $)^{[7 a]}$ or in water with the aid of surfactants or other types of amphiphilic stabilizer ${ }^{[11]}$ However, for many potential applications, stabilizerfree water dispersions of graphene are highly desirable. ${ }^{[4]}$ One way to achieve stable aqueous dispersions from electrochemically exfoliated graphite would be to foster the oxidation reactions that take place during the electrolytic exfoliation of graphite. In this way, the incorporation of different types of oxygen functional groups that enhance the hydrophilicity of graphene sheets would favor their dispersibility in water and lead to the formation of stable colloidal suspensions. On the basis of this hypothesis, we have developed a synthesis strategy directed at obtaining processable aqueous dispersions of graphene from the electrochemical exfoliation of graphite. In preliminary experiments carried out in our laboratory, it was observed that the electrochemical exfoliation of relatively dense pieces of graphite (i.e. rods or sheets) occurs at considerably lower rates than that of the graphite materials (i.e. foil or flakes) that are currently employed in electrochemical exfoliation. The fact that the electrochemical exfoliation of dense pieces of graphite occurs more slowly allows to envisage a considerable enhancement in the incorporation of oxygen groups as consequence of the reactions with the species generated during the oxidation of water, resulting in an improvement in the hydrophilic nature of the exfoliated products. In the present work, we used a graphite rod as anode and a platinum mesh as cathode, while the electrochemical exfoliation was carried out in an aqueous solution of a neutral electrolyte (i.e. ammonium sulfate) at a voltage of $10 \mathrm{~V}$. In order to promote oxidation reactions, the EEG process was prolonged for a relatively large period of time to around $2 \mathrm{~h}$. Intensive sonication of the expanded graphite particles generated during the EEG process gave rise to an 
aqueous dispersion formed by oxidized graphene layers (OG). An aqueous dispersion formed by graphene sheets was easily obtained through the "in-situ" reduction of the dispersed OG layers by applying the procedure reported by Li et al. ${ }^{[3]}$ In this way, a processable and stable aqueous dispersion of graphene sheets was obtained. In order to demonstrate the easy processability of the graphene aqueous dispersion, two types of macroscopic graphene structures were prepared: graphene aerogels and graphene films. These materials were successfully employed as absorbents for removing organic liquids in the case of aerogels and as electrodes in double-layer electrochemical capacitors in the case of the graphene films.

\section{Results and Discussion}

\subsection{Characteristics of the products obtained from the electrochemical exfoliation of graphite}

The electrochemical exfoliation was carried out in a two-electrode cell by using a graphite rod as anode and platinum mesh as cathode, the DC voltage between both electrodes being of $10 \mathrm{~V}$ (see Figure S1 in Supporting Information). In these experiments an aqueous solution of $\left(\mathrm{NH}_{4}\right)_{2} \mathrm{SO}_{4}(0.2 \mathrm{M})$, which has been demonstrated to be an efficient exfoliation salt was used as electrolyte. ${ }^{[8 b]}$ It was observed that $\sim 25$ wt.\% of the starting graphite is consumed due to the oxidation reactions during the EEG process. ${ }^{[7 d]}$ The yield was therefore around 75 wt.\%, which is in agreement with the results reported by other authors. ${ }^{[8 b]}$ In order to prepare a processable colloidal suspension, the expanded graphite produced by the EEG process was sonicated in water, and then subjected to centrifugation to remove the unexfoliated particles. In this way, 
an aqueous dispersion of oxidized graphene-like layers (concentration: $\sim 2-3$ $\mathrm{mg} / \mathrm{mL}$ ) was obtained. The advantage of this dispersion is that it remains stable for at least two months. The percentage of the graphene-like material dispersed in water represents around $30 \mathrm{wt} \%$ of the starting graphite. The synthesis procedure is illustrated in the schemes of Figure 1 and Figure S1.

Three types of products are obtained from the EEG process: a) expanded graphite (EG) particles directly produced by electrochemical exfoliation, b) highly oxidized graphene-like sheets (denoted as OG) that are dispersible in water and c) a residue formed by poorly exfoliated EG particles (denoted as EGR), which was collected by centrifugation. The morphology of these materials was investigated by scanning electron microscopy (SEM). Figures 2a-c show that the EG particles appear to have expanded, with their layers noticeably separated as a consequence of the interlayer forces exerted by the gaseous species (e.g. $\mathrm{O}_{2}, \mathrm{SO}_{2}$, etc.) generated during the EEG process. ${ }^{\left[{ }^{8 b]}\right]}$ Intensive sonication of the EG particles gives rise to the detachment of a large number of graphene-like sheets. It can be reasonably assumed that only those sheets formed by a few graphene layers with a large amount of oxygen functional groups will pass on to the aqueous phase and form a stable dispersion (OG sample). The fact that the OG sheets are easily precipitated by the addition of a certain volume of $\mathrm{NaCl}$ solution suggests that colloidal stability is due to the electrostatic repulsion between the OG sheets (see Figure S1). It seems clear that the repulsive forces are caused by the negatively charged oxygen groups (e.g. carboxylic) (vide infra). It is important to note that the formation of this colloidal aqueous dispersion does not require the use of any foreign chemical species such as surfactants or other types of stabilizer. 
Figures $2 d$-e show SEM images of the OG layers once they have precipitated as a result of the addition of $\mathrm{NaCl}$. These images clearly show the characteristic structure of graphene-like materials, which consists of randomly interconnected graphene-like sheets. Further information about oxidized graphene-like sheets dispersed in water was obtained by means of TEM microscopy. Thus, Figures S2 $(a, b, c)$ show TEM images of flat OG sheets with lateral sizes of $\sim 0.5-1 \mu \mathrm{m}$. Examination of a large number of high-resolution TEM (HRTEM) images evidences that the OG sheets consist of only a few layers, most of which are bilayer. A selection of these HRTEM images is given in Figure 2 ( $f-i)$ and Figure S2 (d-i).

During the EEG process, oxidation reactions occur extensively as a consequence of the attack of hydroxyl and oxygen radicals generated from the anodic oxidation of water on the edge and grain boundary sites of the graphite. ${ }^{[\mathrm{d}]}$ This causes the formation of a large number of oxygen functional groups on the expanded graphite particles. The chemical composition of these samples was determined by elemental analysis. The results presented in Table S1 reveal that they have oxygen contents of around $20 \mathrm{wt.} \%$, with (C/O) atomic ratio values of $4.6,4.5$ and 5.4 for EG, OG and EGR respectively (see Table S1). These results evidence the incorporation of numerous oxygen groups during the EEG process. As expected, the oxidized graphene sheets dispersed in water $(\mathrm{OG})$ have the lowest $(\mathrm{C} / \mathrm{O})$ atomic ratio. This value is higher than the one typical of graphene oxide produced by means of the Hummers' method $(\mathrm{C} / \mathrm{O}<2)$, but it is much lower than that of reduced graphene oxide $(\mathrm{C} / \mathrm{O}$ atomic ratio $>$ 8). In short, on the basis of chemical composition, the OG sample resembles poorly reduced graphene oxide. Thermogravimetric analysis (TGA) 
of the EEG products was carried out under an inert atmosphere up to $800{ }^{\circ} \mathrm{C}$ (Figure S3). The weight losses observed in the $250-400{ }^{\circ} \mathrm{C}$ range are mainly associated with the decomposition of labile oxygen functional groups and they are an indicator of the degree of oxidation. As expected, the OG and ROG samples appear to have experienced the highest and lowest degrees of oxidation, respectively. Further information about the oxygen functional groups present in these materials was acquired from the XPS and ATR-IR spectroscopic analyses. Figure 3a shows the deconvoluted XPS spectra corresponding to the $\mathrm{C}$ 1s peak of the EG and OG samples. They include along with the two dominant components located at $\sim 284.4 \mathrm{eV}\left(\mathrm{C}=\mathrm{C}, \mathrm{sp}^{2}\right.$ hybridized carbon atoms) and at $285.3 \mathrm{eV}$ (C-C, $\mathrm{sp}^{3}$ hybridized carbon atoms), three other additional peaks of lower intensity at higher binding energies which can be attributed to oxygen groups attached to the graphene layers: a peak at $~ 286.6$ $\mathrm{eV}$ associated to C-O groups (hydroxyl and epoxy), a peak at $\sim 288.5 \mathrm{eV}$ assigned to $\mathrm{C}=\mathrm{O}$ carbonyl groups and a peak at $290.6 \mathrm{eV}$ that can be attributed to carboxylic $\mathrm{O}-\mathrm{C}=\mathrm{O}$ groups. As expected, in comparison with the $\mathrm{EG}$ particles, the dispersed OG layers exhibit a higher content in oxygen functional groups (see inset in Figure 3a). Additional evidence of the oxygen functional groups present in the EG and OG samples was obtained by means of ATR-IR spectroscopy. The spectra in Figure 3b allows several characteristic bands to be identified: at $\sim 1060 \mathrm{~cm}^{-1}$ (C-O stretching in ethers or epoxides), between $1300-1460 \mathrm{~cm}^{-1}(\mathrm{O}-\mathrm{H}$ bending vibrations and $\mathrm{C}-\mathrm{OH}$ stretching vibrations from hydroxyl groups), at $1650 \mathrm{~cm}^{-1}$ ( $\mathrm{C}=\mathrm{C}$ skeletal, stretching vibrations from unoxidized graphitic domains) and, in the case of the OG sample, at $\sim 1730 \mathrm{~cm}^{-1}$ ( $\mathrm{C}=\mathrm{O}$ stretching vibrations from carbonyl and carboxylic groups). ${ }^{[12]}$ 
The microstructure of the EEG-based products was investigated by X-ray diffraction and Raman spectroscopy. The XRD patterns in Figure 3c reveal that the EG sample exhibits two characteristic peaks, a broad band at $\sim 11.4^{\circ}(d-$ spacing: $0.78 \mathrm{~nm}$ ) which is ascribable to the interlayer separation due to the oxygen functional groups attached to the graphene sheets ${ }^{[13]}$ and a well-defined peak at around $26.1^{\circ}\left(d_{002}\right.$-spacing: $\left.0.34 \mathrm{~nm}\right)$, at a position identical to that of raw graphite, which suggests that the EG sample still contains a certain number of graphite-like particles. By contrast, the film obtained by filtration of the OG dispersion exhibits a much smaller peak at $23.7^{\circ}$, with a lattice spacing of $\sim 0.38$ $\mathrm{nm}$, which suggests the presence of oxygen functional groups between the graphene layers. The low intensity of this peak indicates that the OG layers will not restack back to graphite when they form a compressed film, but will give rise to a disordered structure typical of graphene-based films. ${ }^{[14]}$ Further evidence of the structural characteristics and defects of the products derived from the EGG process was obtained by means of Raman spectroscopy. Figure $3 d$ shows the first-order region (1000-2000 $\left.\mathrm{cm}^{-1}\right)$ of the Raman spectra of the raw graphite and the products derived from the EGG process. The spectra exhibit three characteristic peaks, which can be associated to two characteristic structural properties. Thus, the $D$ band at $\sim 1350 \mathrm{~cm}^{-1}$ and the $\mathrm{D}^{\prime}$ shoulder at $\sim 1620 \mathrm{~cm}^{-1}$ reflect the presence of defects and structural disorder in the $\mathrm{sp}^{2}$ carbon network. On the other hand, the so called graphitic $\mathrm{G}$ band at $\sim 1590 \mathrm{~cm}^{-1}$ corresponds to the in-plane vibrations of the graphene lattice and it is the only peak in defectfree graphite/graphene materials. In order to evaluate the degree of defects, we used the integrated intensity ratio of the $D$ and $G$ bands $\left(I_{D} / I_{G}\right.$ ratio), whose values are included in Figure $3 \mathrm{~d}$. As might be expected, the raw graphite has a 
very low $I_{D} / I_{G}$ value of 0.1 , reflecting its highly ordered structure. By contrast, the samples produced by the EEG process (e.g. EG and OG) show high values of $I_{D} / I_{G}$ that reveal the presence of significant structural defects in the carbon lattice, which can be ascribed to the reduction in size of the in-plane $\mathrm{sp}^{2}$ domains as a result of the oxidation processes. ${ }^{[15]}$

\subsection{Processable aqueous graphene colloidal suspensions from electrochemically exfoliated graphite}

In 2008, Li et al. demonstrated that is possible to produce stable and processable colloidal suspensions of graphene sheets dispersed in water by means of the reduction of a diluted aqueous dispersion of graphene oxide with hydrazine in the presence of ammonia. ${ }^{[3]}$ In the present work, we have adopted a similar strategy and demonstrate the viability of the production of stable aqueous dispersions of graphene based on the EEG process. Our experiments reveal that the application of the Li's procedure to a diluted dispersion of $O G$ sheets $(0.5 \mathrm{mg} / \mathrm{mL})$ can produce a colloidal suspension of graphene sheets that we have identified as reduced oxidized graphene and denoted as ROG. The resulting dispersion of graphene sheets has a concentration of around 0.3-0.4 $\mathrm{mg} / \mathrm{mL}$. Due to the reduction reactions, a large number of O-groups disappear and, in consequence, the $(\mathrm{C} / \mathrm{O})$ atomic ratio increases from 4.5 for $\mathrm{OG}$ to 11.7 for ROG (see Table S1), a typical value for graphene materials obtained by the chemical reduction of graphene oxide. ${ }^{[16]}$ The reduction process partially restores the electronic conjugation of the graphene layers as evidenced by the UV-vis spectra in Figure 4, which show in the case of the reduced sample an increase in absorbance over the whole wavelength range and a displacement of the absorption peak ( $\pi-\pi^{*}$ transitions of aromatic C-C bonds) from $267 \mathrm{~nm}$ for 
OG to $274 \mathrm{~nm}$ for ROG. ${ }^{[17]}$ The spectrum for a dispersion of graphene oxide (GO) which shows an absorption peak at $230 \mathrm{~nm}$ is included in Figure 4 as a reference. As in the case of $\mathrm{OG}$, the addition of drops of $\mathrm{NaCl}$ solution to the ROG dispersion instantaneously causes it to coagulate (see inset in Figure 4). This shows that the stabilization of the colloidal suspension of ROG is caused by electrostatic repulsion forces between the graphene sheets. Like the dispersed graphene sheets produced by the reduction of graphene oxide ${ }^{[3]}$ the stability of the ROG colloidal suspension is due to the negatively charged oxygen groups (i.e. carboxylic groups attached to the graphene layers) that remain after the reduction reaction. The presence of carboxylic groups in the ROG sample is evidenced by the XPS and ATR-IR spectra in Figures $3 a$ and $3 b$ respectively. Thus, the data in Figure $3 a$ (inset) reveal that, although the reduction of OG involves a notable drop in the number of C-O groups (hydroxyl and epoxy, peak at $\sim 286.6 \mathrm{eV}$ ) and $\mathrm{C}=\mathrm{O}$ carbonyl groups (peak at $\sim 288.5 \mathrm{eV}$ ), the amount of carboxylic $\mathrm{O}-\mathrm{C}=\mathrm{O}$ groups $(290.6 \mathrm{eV})$ hardly varies. This result is corroborated by the ATR-IR spectrum corresponding to the ROG sample, which shows a sharp peak at $1730 \mathrm{~cm}^{-1}$ that can be attributed to $\mathrm{C}=\mathrm{O}$ stretching vibrations from carbonyl and carboxylic groups (Figure 3b). It should also be pointed out that when the ROG dispersion was centrifuged at $9000 \times \mathrm{g}$ for $10 \mathrm{~min}$, $<20$ wt.\% of the graphene sheets precipitated, confirming the stability of the colloidal suspension.

The SEM images of the ROG sample (precipitated from the dispersion with the aid of $\mathrm{NaCl}$ ) show that like the OG sample, it consists of randomly interconnected graphene sheets (Figures 5a-c). Also as in the case of the OG sample, the graphene ROG sheets are flat and have a lateral size of around 1 
$\mu \mathrm{m}$, as can be seen from by the TEM images in Figures $5 d$ and S4 (a-c). The HRTEM images obtained for a large number of ROG sheets show that they consist of one layer or just a few layers (<7), two-layer sheets being the most common, which is consistent with the results obtained for the OG sample (Figures $5 f-i$ and Figures $S 4 d-i)$. This result agrees with the observations of other authors describing graphene sheets obtained from the electrochemical exfoliation of graphite flakes. ${ }^{[7 d, 8 b, 18]}$

The structural characteristics of the ROG sample were evaluated by means of X-ray diffraction and Raman spectroscopy. The XRD pattern corresponding to a ROG film (graphene paper obtained by filtration) exhibits a low intensity peak at $23.9^{\circ}\left(\mathrm{d}_{\text {spacing }} \sim 0.37 \mathrm{~nm}\right)$ which denotes a certain restacking (Figure 3c). On the other hand, the Raman spectrum in Figure $3 d$ reveals that the $\left(I_{D} / I_{G}\right)$ ratio is slightly smaller for ROG $\left(I_{D} / I_{G}=1.61\right)$ than for OG $\left(I_{D} / I_{G}=1.76\right)$, which suggests a certain decrease in the number of defects as a consequence of the removal of oxygen groups.

In order to demonstrate the processability of the ROG aqueous dispersion, we used it to prepare two types of macroscopic graphene structures: a graphene aerogel (GA) and a graphene film. In the following sections, we shall discuss the characteristics of both types of materials and their potential as adsorbents and as electrodes in supercapacitors.

\subsection{EEG-based graphene aerogels as efficient sorbents for organic liquids}

A graphene aerogel (GA) was fabricated from the RGO dispersion by means of a freeze-drying process that involves its rapidly being frozen by being 
quenched in liquid nitrogen followed by a lyophilization step. The as-obtained GA exhibits an ultralow density of around $1 \mathrm{mg} / \mathrm{cm}^{3}$ (porosity $>99 \%$ ) which is one of the lowest density values recorded for carbon aerogels, ${ }^{[19]}$ including graphene-based aerogels obtained by the reduction of graphene oxide foams. ${ }^{[20]}$ An image of one such aerogel is shown in Figure 6a. As expected, this material has a very open structure made up of interconnected graphene nanoscrolls and wrinkled graphene sheets, as illustrated by the SEM images in Figures 6b-d.

The graphene aerogel is highly hydrophobic, as can be deduced by the wide contact angle $\left(\sim 140^{\circ}\right)$ observed for a drop of water on the surface of the GA (see Figure 7a). By contrast, when a drop of organic solvent such as cyclohexane is added to the GA, it is rapidly absorbed (see Figure 7b). Figure 7c illustrates the ability of a piece of GA to remove a layer of pump oil (dyed with Red Oil O) floating on the surface of water. We evaluated the absorption efficiency of GA (defined as weight gain percentage per unit weight of GA) for a variety of organic liquids including organic solvents and oils (Figure 7d). It can be seen that GA has an excellent absorption capacity and is able to absorb organic substances up to 35 to 90 times its own weight, which is in the range of the values measured for graphene foams produced by the reduction of GO obtained by the Hummers method. ${ }^{[21]}$

\subsection{EEG-based free-standing graphene films as electrodes for double layer electrochemical capacitors}

Free-standing graphene films were easily prepared by the vacuum filtration of RGO aqueous dispersions. The filtration process takes place rapidly (i.e. $<30 \mathrm{~min}$ ). In this way, a solvated graphene film with a thickness of around $100 \mu \mathrm{m}$ and an areal density of $\sim 1 \mathrm{mg} / \mathrm{cm}^{2}$ is obtained (see Figure $8 \mathrm{a}$ ). When 
the solvated film is dried, a drastic reduction in the thickness of around $93 \%$ is registered, giving rise to a lustrous flexible graphene paper (see Figure 8b) with a compact layered structure (see Figure 8c) that has a thickness of $\sim 7 \mu \mathrm{m}$ and a high density of $\sim 1.4 \mathrm{~g} / \mathrm{cm}^{3}$. The SEM image in Figure $8 \mathrm{~d}$ shows a detail of the surface of the graphene paper. In general, free-standing graphene films have several important properties that allow the graphene to be advantageously used not only in applications related to the fabrication of flexible energy storage devices (i.e. Li-ion batteries and supercapacitors), ${ }^{[22]}$ but also as sensors and scaffolds for medical and electronic applications. ${ }^{[23]}$ Until now, the fabrication of free-standing graphene films from electrochemically exfoliated graphite has been carried out by the vacuum-filtration of graphene layers dispersed in organic solvents such as $\mathrm{DMF}^{[8 \mathrm{a}]}$ or ethanol. ${ }^{[18 \mathrm{a}]}$ However, to the best of our knowledge, there have been no reports of the preparation of free-standing graphene films from aqueous dispersions produced from electrochemically exfoliated graphite.

The solvated free-standing graphene films produced in this work were used to build up a two-electrode supercapacitor system. The capacitive performance of the device was investigated by means of electrochemical impedance spectroscopy (EIS), cyclic voltammetry (CV) and galvanostatic charge/discharge measurements. As a first step, EIS analysis was used to evaluate the ion diffusion dynamics within the solvated graphene films. Figure 9a compares the frequency response of capacitance in two aqueous electrolytes, the widely used $\mathrm{H}_{2} \mathrm{SO}_{4}$ and the user-friendly alternative $\mathrm{Li}_{2} \mathrm{SO}_{4}$. Even though the capacitance response was slower in $\mathrm{Li}_{2} \mathrm{SO}_{4}$ than in $\mathrm{H}_{2} \mathrm{SO}_{4}$, the relaxation time constant was still very low (i.e. $436 \mathrm{~ms}$ ), suggesting the high rate 
capability of these graphene films in the more convenient $\mathrm{Li}_{2} \mathrm{SO}_{4}$. This is confirmed by the CVs in Figure 9b, which show ideal capacitive behavior up to a scan rate of $2 \mathrm{~V} \mathrm{~s}^{-1}$, with an electrode areal density of $36 \mathrm{mF} \mathrm{cm}^{-2}$ (measured at $0.8 \mathrm{~V})$. Further evaluation of the supercapacitor performance by means of galvanostatic charge/discharge cycling indicates a capacitance retention of 60 $\%$ for a 1000-fold increase in discharge current (from 0.2 to $200 \mathrm{~mA} \mathrm{~cm}{ }^{-2}$ ), as depicted in Figure 9c. In spite of the moderate capacitance of this material (e.g. $44 \mathrm{mF} \mathrm{cm}-2$ at $0.2 \mathrm{~mA} \mathrm{~cm}^{-2}$ ), the large cell voltage of $1.6 \mathrm{~V}$ (see Figure S5a) endows the supercapacitor with a maximum areal energy density of ca. $15 \mu \mathrm{Wh}$ $\mathrm{cm}^{-2}\left(0.25 \mathrm{~mW} \mathrm{~cm}^{-2}\right)$ and still delivers ca. $5 \mu \mathrm{Wh} \mathrm{cm} \mathrm{cm}^{-2}$ at a high power density of $\sim 287 \mathrm{~mW} \mathrm{~cm}^{-2}$ (see Figure S5b). Stability cycling tests show a decrease in capacitance of $8 \%$ during the first 5000 cycles at $5 \mathrm{~A} \mathrm{~g}^{-1}$, but only $3 \%$ after another 5000 cycles (see Figure 9d), confirming the robustness of these graphene films.

\section{Conclusions}

In summary, we report an electrochemically assisted process for producing stable dispersions of graphene in water in three simple steps: (a) the electrochemical exfoliation of graphite, (b) sonication to separate the oxidized graphene layers and (c) the reduction of the oxidized graphene to graphene. The dispersed graphene sheets have a C/O atomic ratio of 11.7 and are made up of only a few graphene layers, though bilayer sheets are the most abundant. Around $30 \mathrm{wt} \%$ of the initial graphite is converted to graphene, which forms stable aqueous dispersions in concentrations of around $2-3 \mathrm{mg} / \mathrm{mL}$. The processability of the aqueous dispersions of graphene allows them to be used directly for fabricating a variety of macroscopic graphene structures. For 
example, graphene aerogels with a high absorbent capacity and graphene films for use as electrodes in supercapacitors can be easily prepared from these graphene dispersions. The process reported here represents an alternative against the classical chemical exfoliation methods (e.g. the Hummers' method), which are complex, harmful and dangerous. 


\section{Experimental Section}

Electrochemical exfoliation of graphite. A graphite rod (Mersen, $99.995 \%$ purity, $3.05 \mathrm{~mm}$ diameter) was used as working electrode connected to the anode for electrochemical exfoliation. A platinum mesh was used as counterelectrode (cathode) and placed parallel to the anode at a distance of around 2 $\mathrm{cm}$. An aqueous solution of ammonium sulphate (Alfa-Aesar) with a concentration of $0.2 \mathrm{M}$ was used as electrolyte. The electrochemical exfoliation process was carried out by applying a preliminary DC voltage of $2 \mathrm{~V}$ for 2 min in order to wet the graphite electrode, as recommended by Ambrosi and Pumera. [8d] Then, a positive DC voltage of $10 \mathrm{~V}$ was applied to the graphite electrode using DC power (Promax, DC-405) for the time required to attain the complete exfoliation of the graphite rod $(\sim 2 \mathrm{~h})$. This process was carried out under stirring, taking care to keep the reaction temperature within the $20-30^{\circ} \mathrm{C}$ range. The expanded graphite particles (EG) detached from the rod during the electrochemical exfoliation were collected by filtration and washed with abundant DI water. Immediately after the washing step, the EG sample was dispersed in water by sonication at high power (Sonics, Ultrasonic Vivracell, 750 $\mathrm{W}, 40 \%)$ for $1.5 \mathrm{~h}$. Afterwards, the sample dispersed in water was centrifuged at a speed of $3000 \mathrm{rpm}(4300 \mathrm{~g})$ for $15 \mathrm{~min}$ to obtain a precipitated solid (REG) and a supernatant dispersion. The aqueous dispersion was formed by oxidized graphene (OG) sheets in concentrations of $\sim 2-3 \mathrm{mg} / \mathrm{mL}$. Figure $\mathrm{S} 1$ shows the experimental setup employed in these experiments.

Processable graphene dispersion. In a typical preparation of an aqueous graphene colloidal dispersion (ROG), $120 \mathrm{~mL}$ of OG dispersion $(0.5 \mathrm{mg} / \mathrm{mL})$ was mixed with $0.24 \mathrm{~mL}$ hydrazine (35 wt.\% in water, Aldrich) and $0.42 \mathrm{~mL}$ 
ammonia (30 wt.\%, Aldrich), stirred for $15 \mathrm{~min}$ and then treated at $100^{\circ} \mathrm{C}$ for $3 \mathrm{~h}$ in a Teflon autoclave.

Preparation of graphene aerogels (GA). In a typical procedure to obtain a GA sample, $120 \mathrm{~mL}$ of ROG dispersion (concentration $\sim 0.35 \mathrm{mg} / \mathrm{mL}$ ) contained in a Teflon vessel was immersed in nitrogen liquid in order to ensure a rapid freezing rate. The frozen dispersion was then transferred to a lyophilizer (Telstar Cryodos) and freeze-dried at a temperature of $-51{ }^{\circ} \mathrm{C}$ and at a pressure of 0.06 mbar. In this way, a graphene aerogel (density $\sim 1 \mathrm{mg} \mathrm{cm}^{-3}$ ) was obtained.

Preparation of graphene films. To prepare the graphene film, around 30 $\mathrm{mL}$ of aqueous ROG dispersion was vacuum filtered through a mixed cellulose ester membrane (0.22 $\mu \mathrm{m}$ pore size, $47 \mathrm{~mm}$ diameter, Millipore). Once the filtration was completed (after $\sim 20 \mathrm{~min}$ ), the solvated film was immediately immersed in water where it was stored. To obtain a dry graphene film (graphene paper), the solvated film was transferred to ethanol for several hours and then it was placed on a glass surface where it was left to dry at room temperature.

Material Characterization: Scanning electron microscopy (SEM) images were obtained on a Quanta FEG650 (FEI) instrument, whereas transmission electron microscopy (TEM) images were recorded on a JEOL (JEM 2100-F) apparatus operating at $200 \mathrm{kV}$. X-ray diffraction (XRD) patterns were obtained on a Siemens D5000 instrument operating at $40 \mathrm{kV}$ and $20 \mathrm{~mA}$, using $\mathrm{Cu} \mathrm{KR}$ radiation. The Raman spectra were recorded on a Horiva (LabRam HR-800) spectrometer. The source of radiation was a laser operating at a wavelength of $514 \mathrm{~nm}$ and at a power of $25 \mathrm{~mW}$. X-ray photoelectron spectroscopy (XPS) was 
carried out on a Specs spectrometer, using Mg KR (1253.6 eV) radiation emitted from a double anode at $150 \mathrm{~W}$. UV-Vis absorption spectra were recorded on a spectrophotometer (Shimadzu UV-2401PC). ATR-FTIR spectra were obtained on a Nicolet 3700 spectrometer (Thermo Scientific) using diamond as ATR crystal. The elemental analysis ( $\mathrm{C}, \mathrm{H}, \mathrm{N}$ and $\mathrm{O}$ ) of the samples was carried out on a LECO CHN-932 microanalyzer.

Absorption Experiments. The capacity of the graphene aerogel to absorb different organic solvents was determined by weight measurements. Weighed pieces of graphene aerogel $(\sim 5-7 \mathrm{mg})$ were immersed in different organic liquids and taken out using tweezers after 30 seconds. After removing the excess organic liquid from the surface with a cellulose filter paper, the samples were weighed again. The amount of organic liquid absorbed was calculated from the differences in mass.

Characterization of the electrochemical supercapacitors: The electrochemical measurements were performed in a two-electrode Swagelok ${ }^{\mathrm{TM}}$ type cell using $1 \mathrm{M} \mathrm{Li}_{2} \mathrm{SO}_{4}$ as electrolyte. The electrochemical capacitor was assembled using two graphene papers of comparable mass and thickness, electrically isolated by a glassy fibrous separator. The electrochemical characterization which was performed on a computer-controlled potentiostat (Biologic VMP3 multichannel generator) consisted of cyclic voltammetry experiments, electrochemical impedance spectroscopy studies (EIS) and galvanostatic charge/discharge cycling tests (CD). More details about the electrochemical tests and related calculations can be found in the Supporting Information. 


\section{Supporting Information}

Supporting Information is available from the Wiley Online Library or from the author.

\section{Acknowledgments}

This research work was supported by the FICYT Regional Project (GRUPIN14102), Spanish MINECO (CTQ2015-63552-R) and Fondo Europeo de Desarrollo Regional (FEDER). G. A. F. thanks the MINECO for his predoctoral contract. 


\section{References:}

1. a) M. J. Allen, V. C. Tung and R. B. Kaner, Chem. Rev. 2010, 110, 132-145; b) H.-P. Cong, J.-F. Chen and S.-H. Yu, Chem. Soc. Rev. 2014, 43, 7295-7325.

2. K. R. Paton, E. Varrla, C. Backes, R. J. Smith, U. Khan, A. O’Neill, C. Boland, M. Lotya, O. M. Istrate, P. King, T. Higgins, S. Barwich, P. May, P. Puczkarski, I. Ahmed, M. Moebius, H. Pettersson, E. Long, J. Coelho, S. E. O’Brien, E. K. McGuire, B. M. Sanchez, G. S. Duesberg, N. McEvoy, T. J. Pennycook, C. Downing, A. Crossley, V. Nicolosi and J. N. Coleman, Nat Mater 2014, 13, 624-630.

3. D. Li, M. B. Muller, S. Gilje, R. B. Kaner and G. G. Wallace, Nat Nano 2008, 3, 101-105.

4. $\quad$ C. Cheng and D. Li, Adv. Mater. 2013, 25, 13-30.

5. D. R. Dreyer, S. Park, C. W. Bielawski and R. S. Ruoff, Chem. Soc. Rev. 2010, 39, 228-240.

6. a) C. T. J. Low, F. C. Walsh, M. H. Chakrabarti, M. A. Hashim and M. A. Hussain, Carbon 2013, 54, 1-21; b) L. Niu, J. N. Coleman, H. Zhang, H. Shin, M. Chhowalla and Z. Zheng, Small 2016, 12, 272-293; c) A. Ambrosi, C. K. Chua, N. M. Latiff, A. H. Loo, C. H. A. Wong, A. Y. S. Eng, A. Bonanni and M. Pumera, Chem. Soc. Rev. 2016, 45, 2458-2493.

7. a) C.-Y. Su, A.-Y. Lu, Y. Xu, F.-R. Chen, A. N. Khlobystov and L.-J. Li, ACS Nano 2011, 5, 2332-2339; b) Z. Liu, Z.-S. Wu, S. Yang, R. Dong, X. Feng and K. Müllen, Adv. Mater. 2016, 28, 2217-2222; c) J. Liu, H. Yang, S. G. Zhen, C. K. Poh, A. Chaurasia, J. Luo, X. Wu, E. K. L. Yeow, N. G. Sahoo, J. Lin and Z. Shen, RSC Adv. 2013, 3, 11745-11750; d) K. Parvez, R. Li, S. R. Puniredd, Y. Hernandez, F. Hinkel, S. Wang, X. Feng and K. Müllen, ACS Nano 2013, 7, 3598-3606.

8. a) J. Liu, M. Notarianni, G. Will, V. T. Tiong, H. Wang and N. Motta, Langmuir 2013, 29, 13307-13314; b) K. Parvez, Z.-S. Wu, R. Li, X. Liu, R. Graf, X. Feng and K. Müllen, J. Am. Chem. Soc. 2014, 136, 6083-6091; c) F. Lou, M. E. M. Buan, N. Muthuswamy, J. C. Walmsley, M. Ronning and D. Chen, J. Mater. Chem. A 2016, 4, 1233-1243; d) A. Ambrosi and M. Pumera, Chem. Eur. J. 2016, 22, 153-159.

9. a) J. M. Munuera, J. I. Paredes, S. Villar-Rodil, M. Ayán-Varela, A. Pagán, S. D. Aznar-Cervantes, J. L. Cenis, A. Martínez-Alonso and J. M. D. Tascón, Carbon 2015, 94, 729-739; b) K. Parvez, S. Yang, X. Feng and K. Müllen, Synthetic Metals 2015, 210, Part A, 123-132.

10. S. Yang, M. R. Lohe, K. Müllen and X. Feng, Adv. Mater. 2016, n/a-n/a.

11. J. M. Munuera, J. I. Paredes, S. Villar-Rodil, M. Ayan-Varela, A. MartinezAlonso and J. M. D. Tascon, Nanoscale 2016, 8, 2982-2998.

12. M. J. Fernández-Merino, L. Guardia, J. I. Paredes, S. Villar-Rodil, P. SolísFernández, A. Martínez-Alonso and J. M. D. Tascón, J. Phys. Chem. C 2010, 114, 6426-6432.

13. D. C. Marcano, D. V. Kosynkin, J. M. Berlin, A. Sinitskii, Z. Sun, A. Slesarev, L. B. Alemany, W. Lu and J. M. Tour, ACS Nano 2010, 4, 4806-4814.

14. X. Yang, C. Cheng, Y. Wang, L. Qiu and D. Li, Science 2013, 341, 534-537.

15. S. Stankovich, D. A. Dikin, R. D. Piner, K. A. Kohlhaas, A. Kleinhammes, Y. Jia, Y. Wu, S. T. Nguyen and R. S. Ruoff, Carbon 2007, 45, 1558-1565.

16. S. Gambhir, R. Jalili, D. L. Officer and G. G. Wallace, NPG Asia Mater. 2015, 7, e186. 
17. L. Guardia, M. J. Fernández-Merino, J. I. Paredes, P. Solís-Fernández, S. VillarRodil, A. Martínez-Alonso and J. M. D. Tascón, Carbon 2011, 49, 1653-1662.

18. a) L. Wu, W. Li, P. Li, S. Liao, S. Qiu, M. Chen, Y. Guo, Q. Li, C. Zhu and L. Liu, Small 2014, 10, 1421-1429; b) J. Wang, K. K. Manga, Q. Bao and K. P. Loh, J. Am. Chem. Soc. 2011, 133, 8888-8891.

19. a) Z.-Y. Wu, C. Li, H.-W. Liang, J.-F. Chen and S.-H. Yu, Angew. Chem. Int. Ed. 2013, 52, 2925-2929; b) J. Zou, J. Liu, A. S. Karakoti, A. Kumar, D. Joung, Q. Li, S. I. Khondaker, S. Seal and L. Zhai, ACS Nano 2010, 4, 7293-7302.

20. a) Y. Zhao, C. Hu, Y. Hu, H. Cheng, G. Shi and L. Qu, Angew. Chem. Int. Ed. 2012, 51, 11371-11375; b) H. Sun, Z. Xu and C. Gao, Adv. Mater. 2013, 25, 2554-2560.

21. a) H. Bi, X. Xie, K. Yin, Y. Zhou, S. Wan, L. He, F. Xu, F. Banhart, L. Sun and R. S. Ruoff, Adv. Funct. Mater. 2012, 22, 4421-4425; b) Z. Niu, J. Chen, H. H. Hng, J. Ma and X. Chen, Adv. Mater. 2012, 24, 4144-4150.

22. J. Ji, Y. Li, W. Peng, G. Zhang, F. Zhang and X. Fan, Adv. Mater. 2015, 27, 5264-5279.

23. X. Wang, Z. Xiong, Z. Liu and T. Zhang, Adv. Mater. 2015, 27, 1370-1375. 


\section{List of Figures}

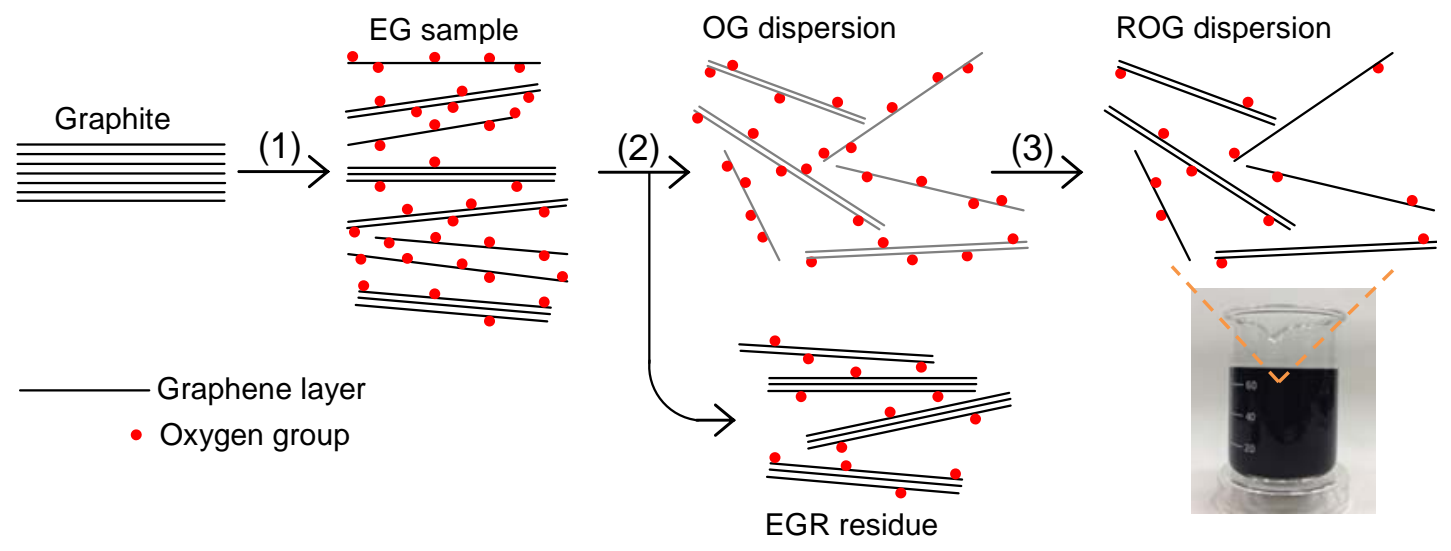

Figure 1. Schematic illustration of the synthesis procedure for obtaining an aqueous dispersion of graphene from electrochemically exfoliated graphite. (1) Electrochemical exfoliation of graphite (EEG process), (2) high-power sonication followed by a centrifugation step to precipitate the un-exfoliated graphene layers (EGR residue) and (3) reduction of the oxidized graphene layers with hydrazine. 


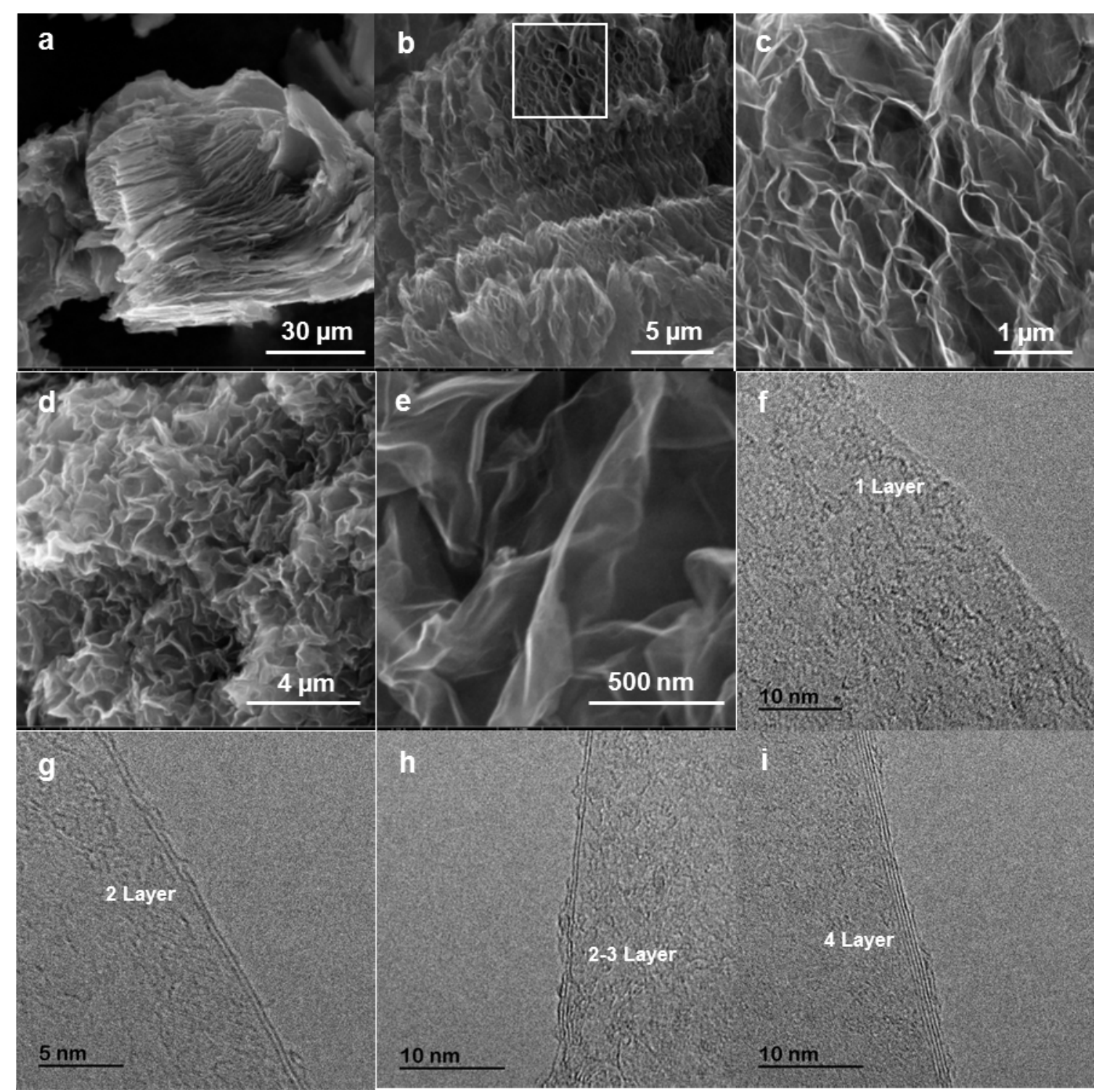

Figure 2. Representative SEM images of the expanded graphite EG (a, b, c) and oxidized graphene-like layers OG $(d, e)$, and high-resolution TEM images of OG sheets comprising different numbers of layers (f, $g, h, i)$. 
a)

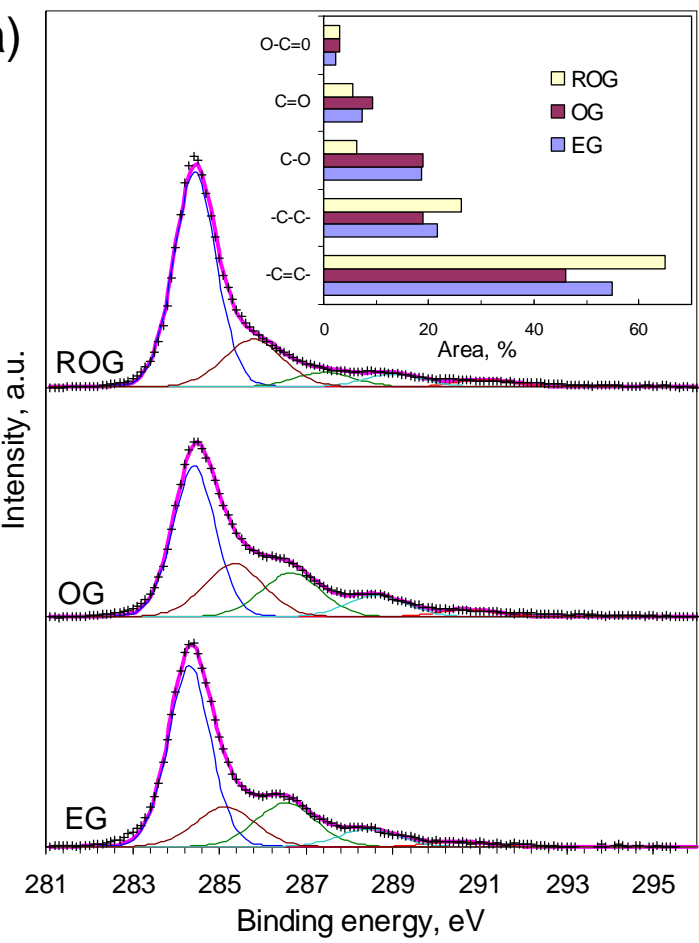

c)

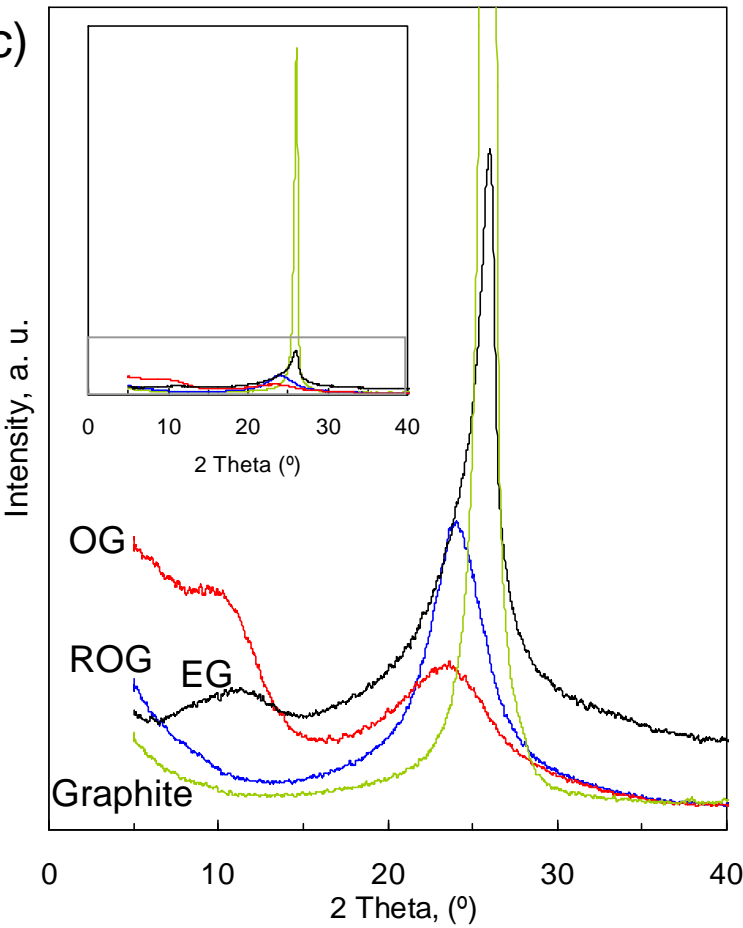

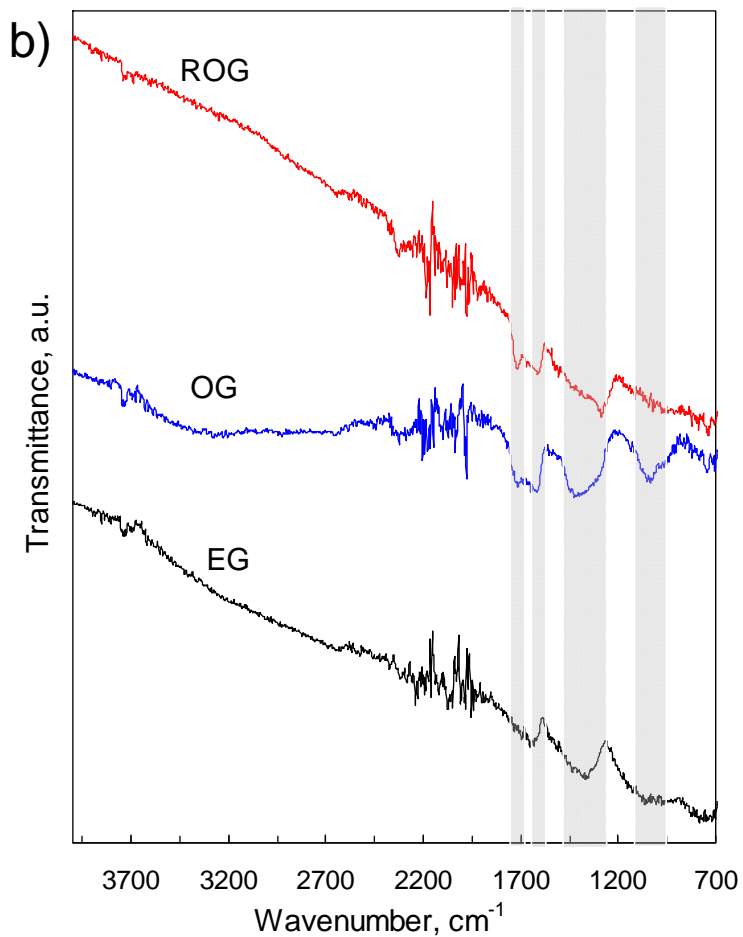

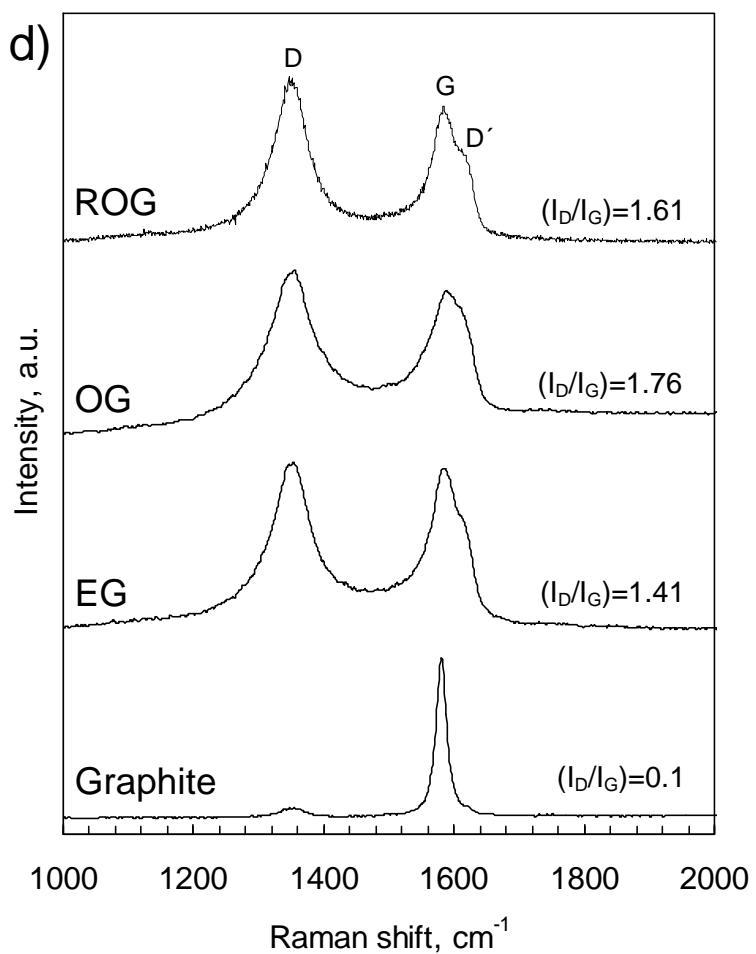

Figure 3. (a) High-resolution core level C1s spectra (Inset shows the percentage (area) of different functional groups identified by deconvolution), (b) ATR-IR spectra of the samples obtained from the EEG process, (c) XRD patterns and (d) normalized Raman spectra. 


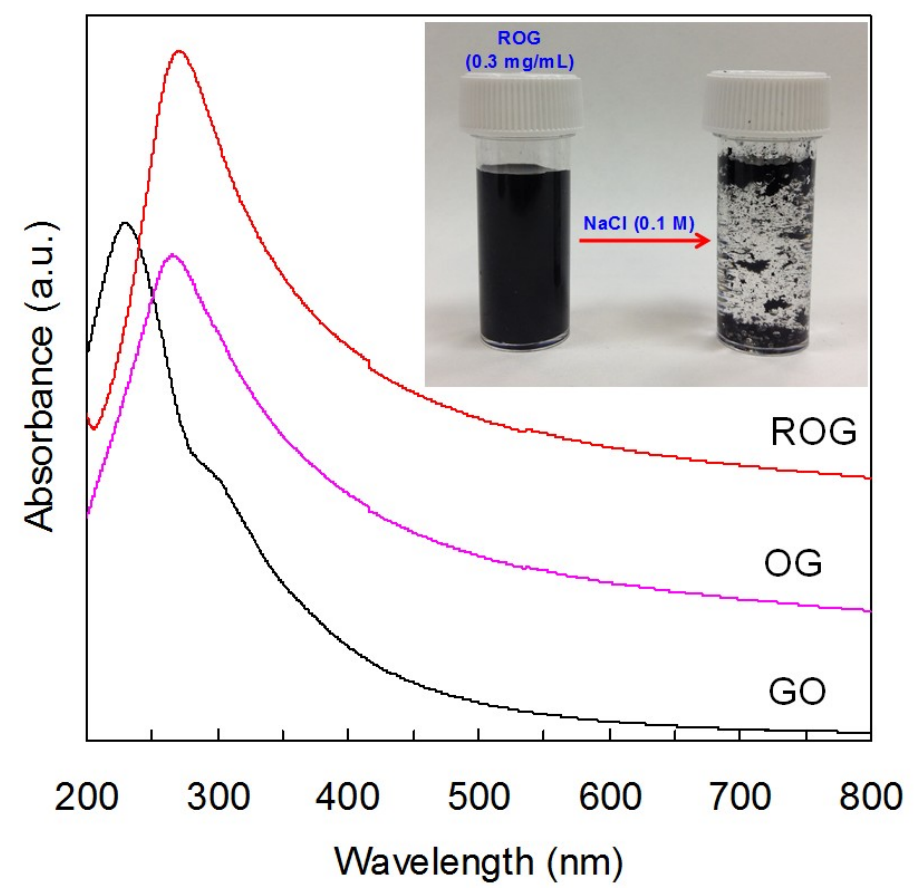

Figure 4. UV-vis absorption spectra of the colloidal suspensions of GO (Graphene oxide obtained by the Hummers' method), OG and ROG. Inset: destabilization (coagulation) of the ROG colloidal suspension by the addition of sodium chloride (salt effect). 


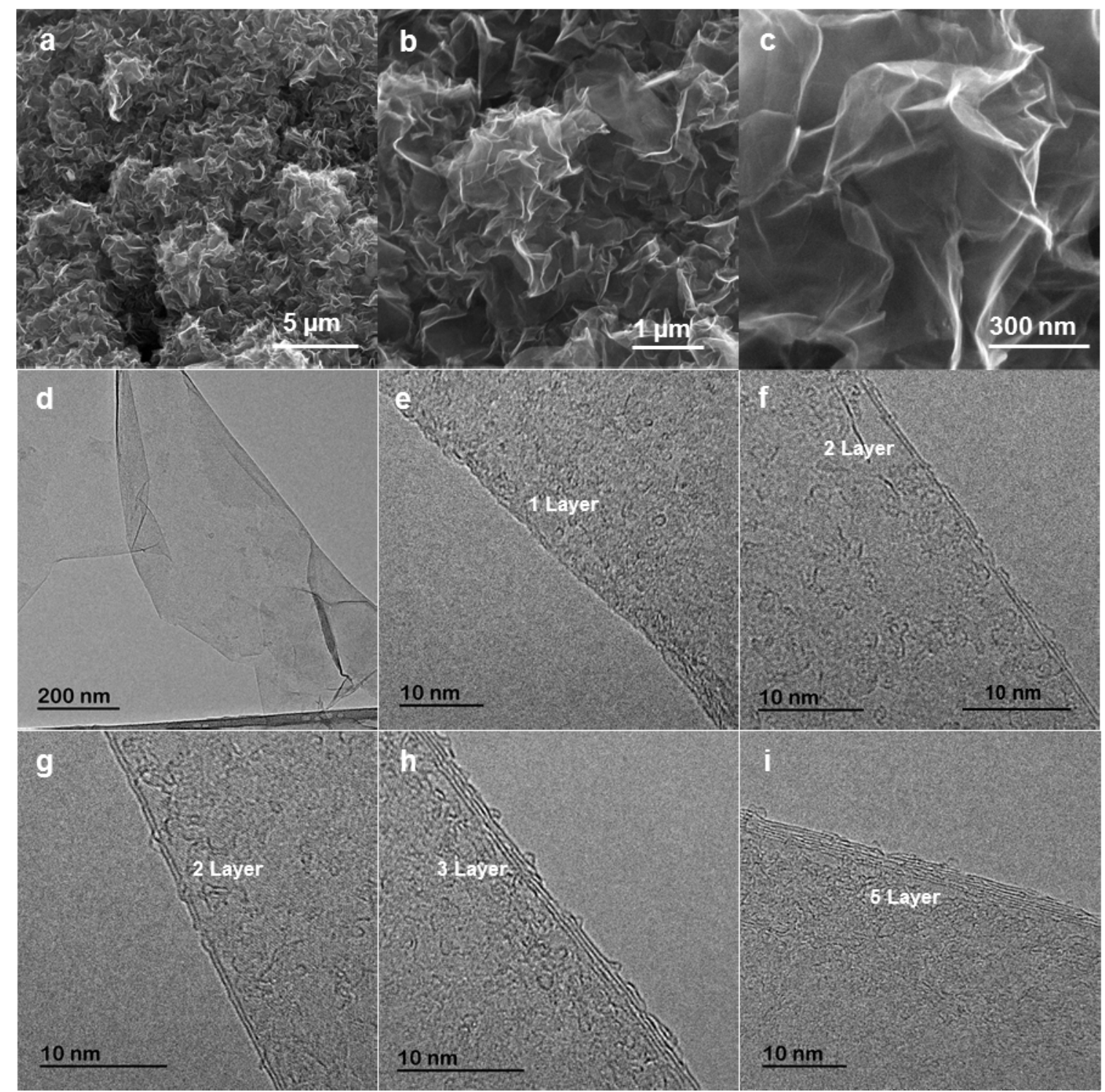

Figure 5. Representative SEM images of the graphene ROG sheets $(a, b, c)$, TEM image of a ROG sheet (d) and high-resolution TEM images of ROG sheets comprising different numbers of layers $(f, g, h, i)$. 

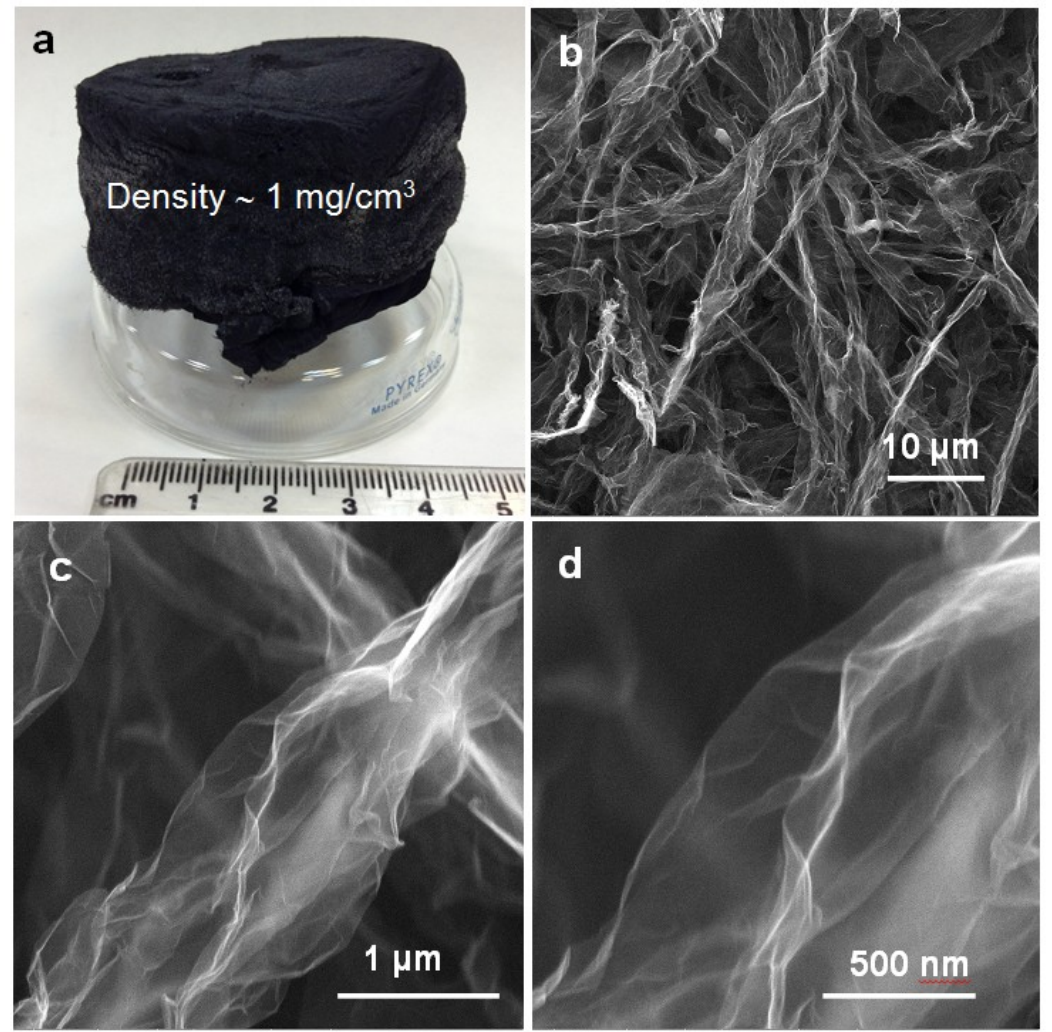

Figure 6. (a) Digital photograph of a graphene aerogel monolith and (b, c, d) SEM images of the structure of the graphene aerogel. 


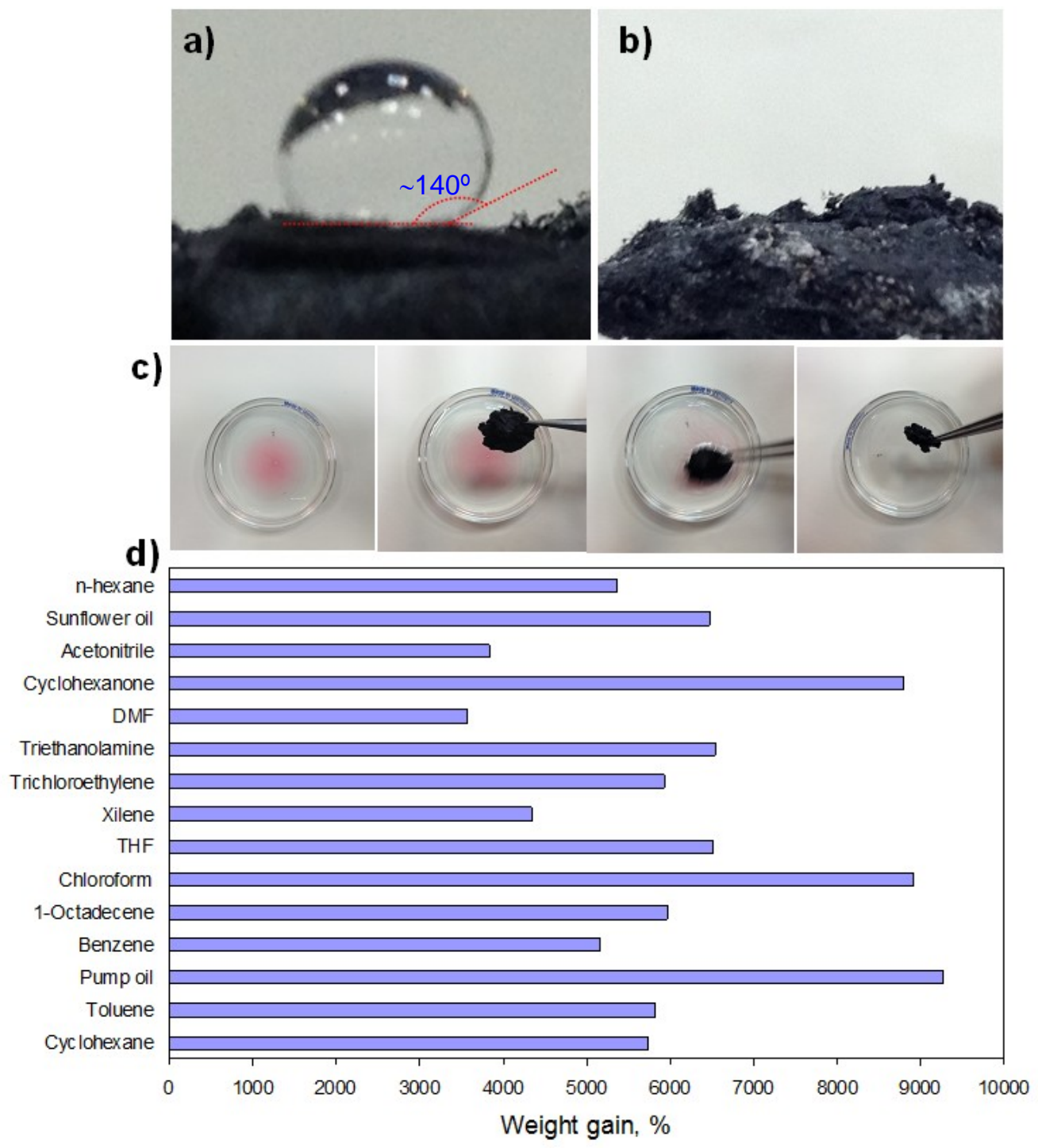

Figure 7. (a) Drop of water on the surface of a graphene aerogel (GA), (b) surface of GA immediately after the addition of a drop of cyclohexane (fast absorption), (c) absorption of pump oil (stained with Red Oil O) by a piece of GA, and (d) absorption efficiency of GA for different organic liquids. 

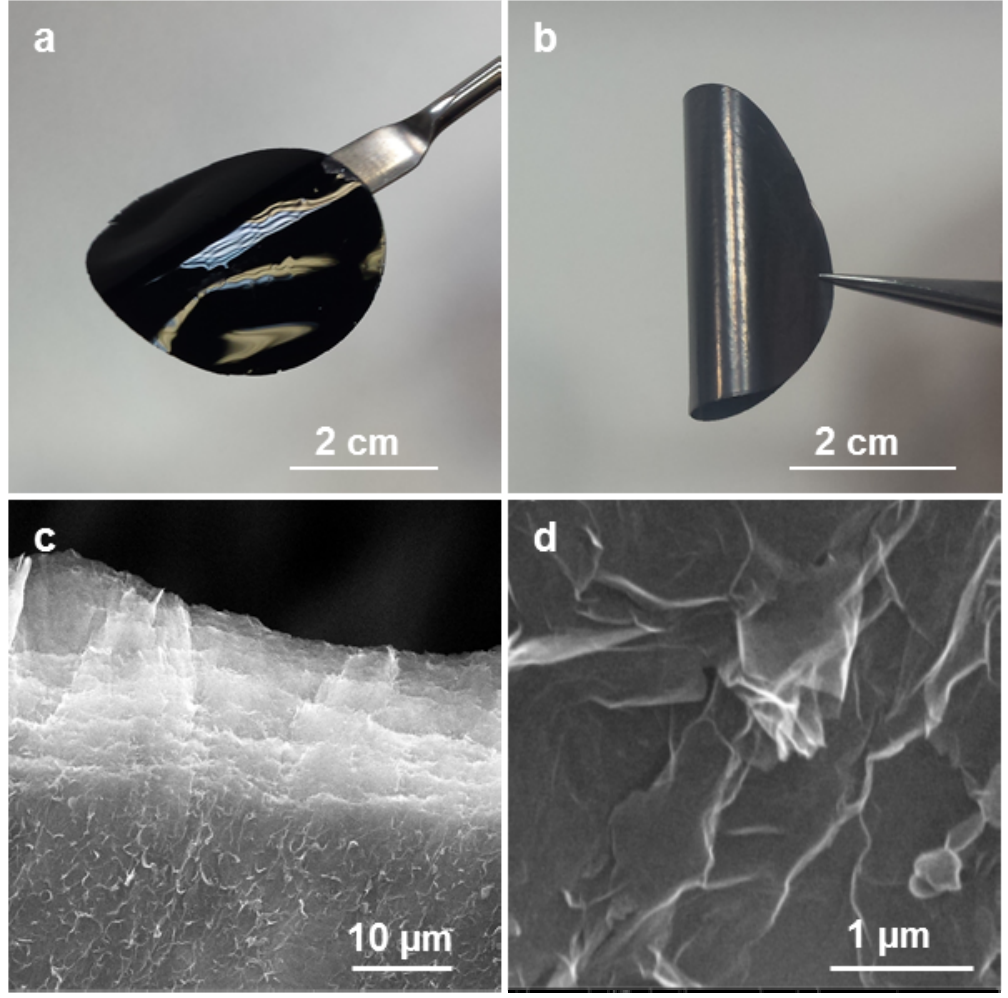

Figure 8. (a) Solvated graphene film, (b) graphene paper, 8c) characteristic cross-section SEM images of the graphene paper and (d) top view SEM image of the graphene paper. 
a)

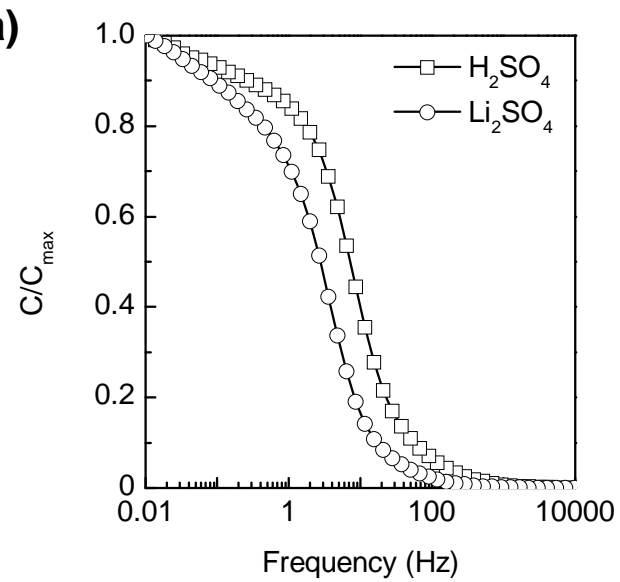

c)

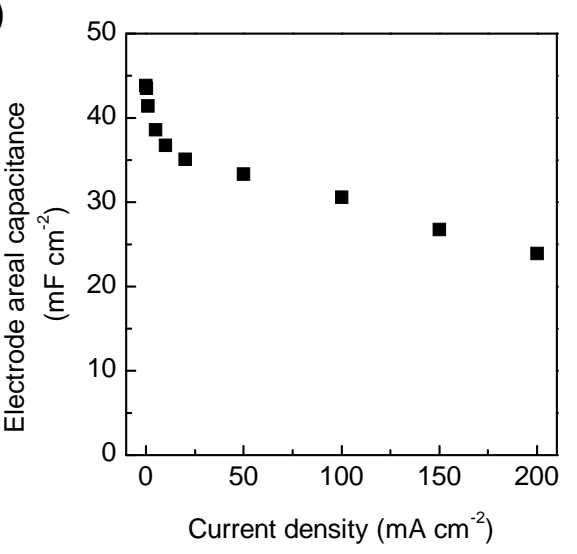

b)

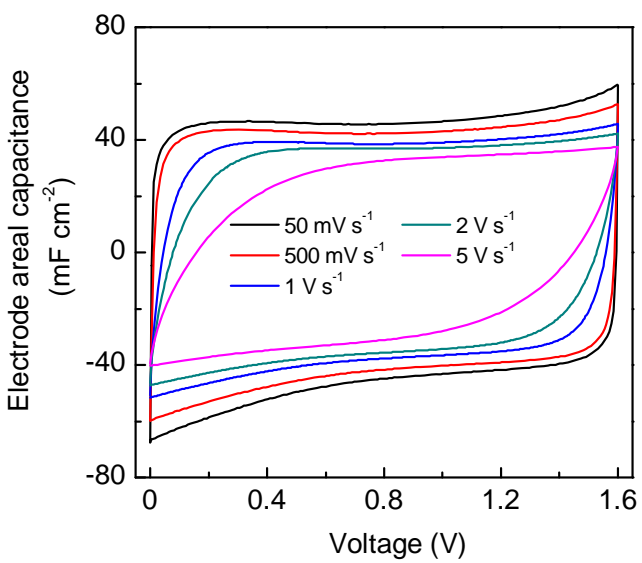

d)

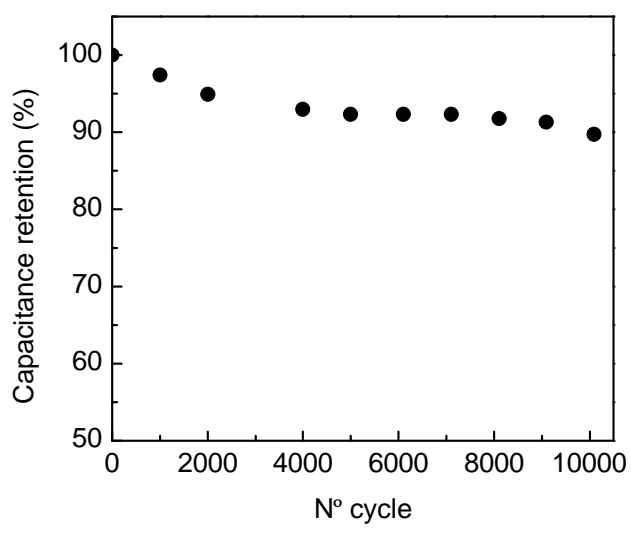

Figure 9. Supercapacitor performance of the solvated graphene films. a) Frequency dependence of capacitance in the $1 \mathrm{M} \mathrm{H}_{2} \mathrm{SO}_{4}$ and $\mathrm{Li}_{2} \mathrm{SO}_{4}$ electrolytes, b) cyclic voltammograms at increasing scan rate of $1 \mathrm{M} \mathrm{Li}_{2} \mathrm{SO}_{4}, \mathrm{c}$ ) impact of the discharge current density on the electrode areal capacitance in 1 $\mathrm{M} \mathrm{Li}_{2} \mathrm{SO}_{4}$ and d) long-term cycling stability at $5 \mathrm{~A} \mathrm{~g}^{-1}$ and $1.6 \mathrm{~V}$. 
Graphical Abstract

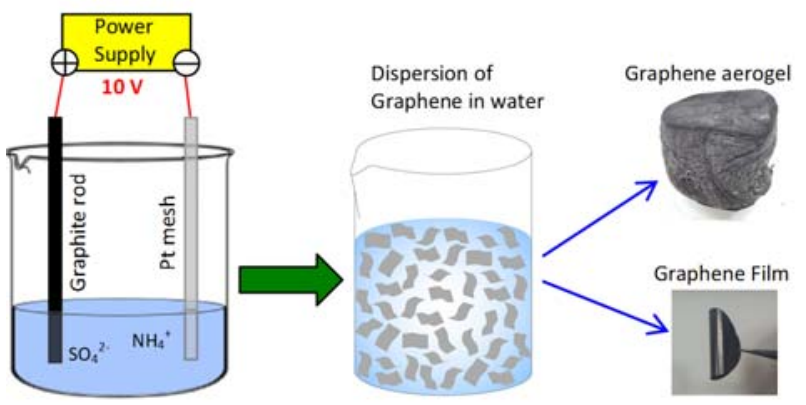

Processable dispersions of graphene in water are important to fabricate a variety of graphene-based products. A facile and high-yield approach is presented to prepare aqueous graphene dispersions based on the electrochemical exfoliation of graphite. The processability of the graphene dispersion has been demonstrated in the fabrication of aerogels and films, which have been employed respectively as absorbents and as electrodes in supercapacitors. 